\title{
Infrared Nanospectroscopy of Phospholipid and Surfactin Monolayer Domains
}

\author{
Bernd Kästner, ${ }^{*} \dagger \odot$ C. Magnus Johnson, ${ }^{\ddagger}$ Peter Hermann, ${ }^{\dagger, \perp}$ Mattias Kruskopf, ${ }^{\S, \#}$ Klaus Pierz, ${ }^{\S}$ \\ Arne Hoehl, ${ }^{\dagger}$ Andrea Hornemann, ${ }^{\dagger}$ Georg Ulrich, ${ }^{\dagger}$ Jakob Fehmel, ${ }^{\dagger}$ Piotr Patoka," Eckart Rühl, ${ }^{\| \odot}$ \\ and Gerhard Ulm ${ }^{\dagger}$ \\ ${ }^{\dagger}$ Physikalisch-Technische Bundesanstalt (PTB), Abbestr. 2-12, 10587 Berlin, Germany \\ ${ }^{\ddagger}$ Department of Chemistry, Division of Surface and Corrosion Science, KTH Royal Institute of Technology, SE-100 44 Stockholm, \\ Sweden \\ ${ }^{\S}$ Physikalisch-Technische Bundesanstalt (PTB), Bundesallee 100, 38116 Braunschweig, Germany \\ "Physikalische Chemie, Institut für Chemie und Biochemie, Freie Universität Berlin, Takustr. 3, 14195 Berlin, Germany
}

\section{Supporting Information}

\begin{abstract}
A main challenge in understanding the structure of a cell membrane and its interactions with drugs is the ability to chemically study the different molecular species on the nanoscale. We have achieved this for a model system consisting of mixed monolayers (MLs) of the biologically relevant phospholipid 1,2-distearoyl-sn-glycero-phosphatidylcholine and the antibiotic surfactin. By employing nano-infrared (IR) microscopy and spectroscopy in combination with atomic force microscopy imaging, it was possible to identify and chemically detect domain formation of the two constituents as well as to obtain IR spectra of these species with a spatial resolution on the nanoscale. A novel method to enhance the near-field imaging contrast of organic MLs by plasmon interferometry is

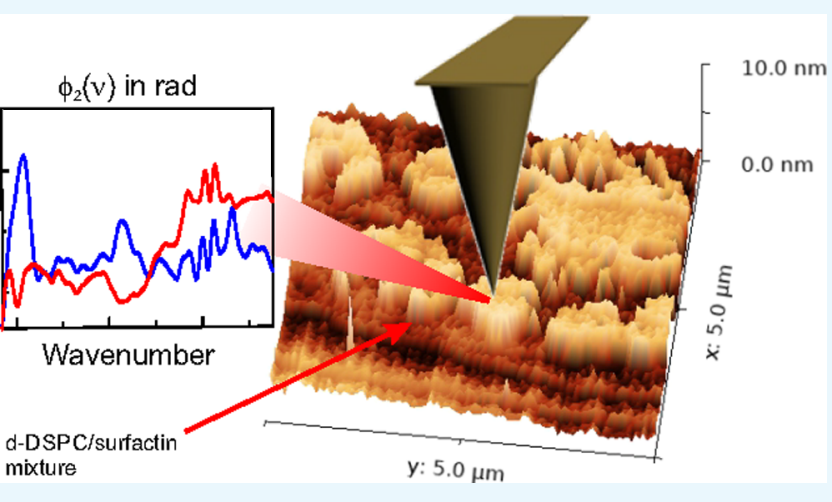
proposed and demonstrated. In this technique, the organic layer is deposited on gold and ML graphene substrates, the latter of which supports propagating surface plasmons. Plasmon reflections arising from changes in the dielectric environment provided by the organic layer lead to an additional contrast mechanism. Using this approach, the interfacial region between surfactin and the phospholipid has been mapped and a transition region is identified.
\end{abstract}

\section{INTRODUCTION}

Cell membranes constitute a barrier around cells toward the surrounding environment and may thus directly interact with drugs distributed in organisms. To obtain a deeper understanding on the uptake of drugs, their interactions with cell membranes are hence of utmost importance. Important and major constituents in cell membranes are phospholipids, which together with, for example, cholesterol and proteins determine the properties of the membranes. These membranes do not consist of a random distribution of phospholipids but rather microscopic domains of disordered character as well as socalled lipid rafts, which possess a high degree of order and commonly contain long-chain saturated phospholipids, sphingolipids, and cholesterol. ${ }^{1-3}$ Drugs are likely to interact with and bind differently to membrane domains of different characters. Therefore, it is of interest to study how and where exactly drug interactions within a cell membrane take place with a spatial resolution on the nanoscale. Moreover, being able to chemically distinguish the nanodomains of different lipids within a biological cell membrane would represent an important step toward the understanding of the structure and functioning of a cell.

Analytical microscopy instruments, such as conventional infrared (IR) microscopy and confocal Raman microscopy, are able to provide spatially resolved chemical information. One major challenge of conducting nanoscale studies of cell membranes is the fact that these approaches have a spatial resolution that is diffraction-limited, yielding some micrometers or a few hundreds of nanometers, depending on the probing wavelength. ${ }^{4}$ In many investigations of biochemical systems, this spatial resolution is not sufficiently detailed ${ }^{5}$ to address the crucial issues mentioned above. Other types of analytical techniques, such as atomic force microscopy (AFM) or electron microscopy, are capable of providing a spatial resolution on the nanoscale but lack the corresponding chemical information.

Received: December 5, 2017

Accepted: March 16, 2018

Published: April 12, 2018 
However, with advanced techniques such as IR nanospectroscopy, it is nowadays possible to obtain chemical information with a spatial resolution of less than $20 \mathrm{~nm} .{ }^{6}$ Of great importance is that this technique is label-free; that is, the intrinsic IR spectra of the cell membranes or the drug are directly employed for their identification, which avoids using additional chemical or biological labels, ${ }^{7}$ such as fluorophores. For the present investigation on phospholipid monolayers (MLs), this is a very important issue, as labels having a typical size corresponding to a significant fraction of the phospholipid are avoided. IR nanospectroscopy has recently been used to probe biomolecules, such as proteins, ${ }^{8-10}$ viruses, ${ }^{8,11,12}$ triglyceride vesicles, ${ }^{13}$ biominerals, ${ }^{14}$ or thiol MLs attached to $\mathrm{Au}$ substrates, ${ }^{15}$ but until now, no studies of (model) phospholipid membranes have been reported. Obtaining spatially resolved IR spectra from a phospholipid layer over a nanoscale region is challenging because the bilayer structure of the phospholipids in a cell membrane has a thickness of only around $5 \mathrm{~nm},{ }^{16}$ and accordingly only a few molecules will contribute to the recorded signal. In addition, the membranes are relatively soft and easily destroyed, especially when AFM is operated in the contact mode.

Biological membranes are complex structures containing numerous kinds of phospholipids, sphingolipids, cholesterol, and proteins. In this study, we have applied IR nanospectroscopy and near-field IR microscopy to identify phase separation in simple supported MLs of the deuterated form of the phospholipid 1,2-distearoyl-sn-glycero-phosphatidylcholine (hereafter denoted as " $d$-DSPC") and the strongly surfaceactive agent surfactin, shown in Figure 1. DSPC was chosen
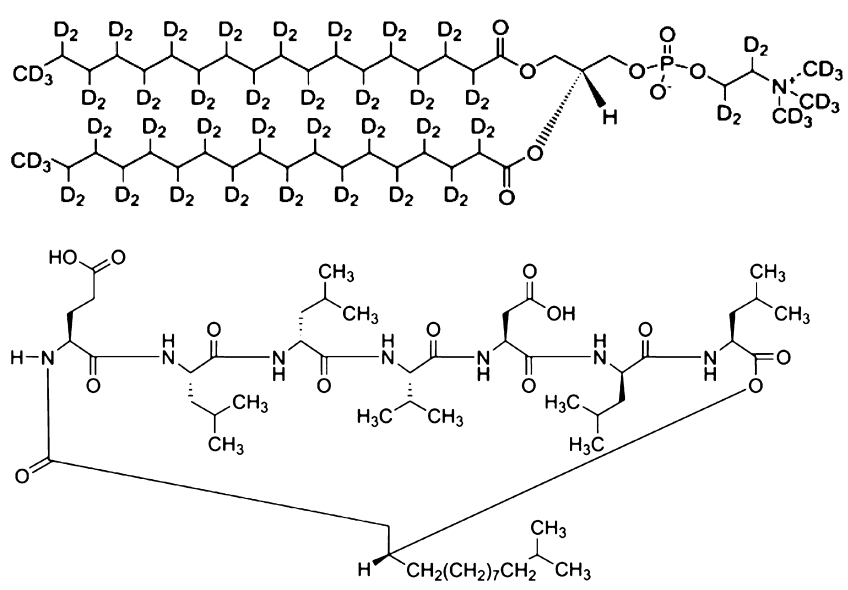

Figure 1. Molecular structures of deuterated DSPC- $d_{83}$ (top) and surfactin (bottom).

because it is a phospholipid abundant in numerous cell membranes, and its deuterated form was used to avoid spectral overlap with the IR-bands originating from the surfactin. As an antibiotic with the ability to penetrate cell membranes and to induce changes in the membrane permeability, surfactin has an important effect on biological systems. ${ }^{17,18}$ Because supported MLs can be studied in air and are easier to prepare than supported bilayers, which require an aqueous environment to remain stable, Langmuir-Blodgett (LB) MLs of the individual species as well as mixtures of the two compounds were prepared. Mixed surfactin/DSPC MLs have been previously studied by AFM, and the results indicated that a phase separation into domains occurred. ${ }^{19}$ However, because AFM cannot provide chemical information, the composition of these domains is still unknown. In this work, we combine near-field IR microscopy, synchrotron-based IR nanospectroscopy, ${ }^{20-23}$ and topographical mapping by AFM to investigate the chemical composition of these domains. In general, being able to chemically distinguish nanodomains in (model) cell membranes is an important step toward a deeper knowledge about domain formation in biological cell membranes as well as how cell membranes interact with pharmaceuticals at a molecular level.

\section{EXPERIMENTAL SECTION}

For the preparation of the supported MLs of $d$-DSPC and surfactin, a Langmuir trough (KSV 5000 LB, Biolin Scientific, Sweden) was used. The two molecules were dissolved in chloroform $(\sim 1 \mu \mathrm{mol} / \mathrm{mL})$, and a few droplets of the solution were gently placed on the water surface in the Langmuir Teflon trough. Two Delrin barriers were compressed to a surface pressure of $25 \mathrm{mN} / \mathrm{m}$ (after evaporation of the chloroform), which is close to the surface pressure experienced by the phospholipids in cell membranes. ${ }^{24}$ The substrates initially located under the water surface were then lifted at a speed of $1 \mathrm{~mm} / \mathrm{min}$, which resulted in the formation of a LB ML on the substrate. In the $d$-DSPC/surfactin mixtures, $75 \mathrm{~mol} \% d$-DSPC and $25 \mathrm{~mol} \%$ surfactin were mixed and spread on the water surface. Adsorbing a lipid ML onto a solid substrate could affect the packing behavior in comparison to the state on the water surface. However, the molecular structure and, for example, domain formation, as is of interest to this article, are believed to be preserved when transferring the lipid ML from the water surface.

The MLs were prepared on two different substrates. The first one was a commercial gold-coated glass coverslip (Pelco), leading to strong signal enhancement in the IR nanospectroscopy measurements. The second type of substrate was $\mathrm{ML}$ graphene on silicon carbide $(\mathrm{SiC})$. Graphene has been used in the past in combination with IR nanospectroscopy, such as for enhancing the resolution in subsurface imaging ${ }^{25,26}$ or for measurements in an aqueous environment. ${ }^{11}$ The employed method for the epitaxial growth of graphene on $6 \mathrm{H}-\mathrm{SiC}(0001)$ is described in ref 27.

The IR nanospectroscopy measurements and near-field imaging were performed on a scattering-type scanning nearfield optical microscope (s-SNOM) (Neaspec GmbH, Germany) consisting of an AFM system and an asymmetric Michelson interferometer for phase-resolved IR nanospectroscopy, which is called, in the following, nano-Fourier-transform (FT) IR spectroscopy. The setup is described in more detail in ref 28. The AFM system was operated in the tapping mode, and the tip oscillated with an amplitude $\Delta z$ just above the sample surface at a frequency $\Omega$. Typical values for the measurements discussed in this work are $\Delta z=80-100 \mathrm{~nm}$. The Au-coated Si cantilevers (Nanosensors PPP-NCSTAu, NanoAndMore $\mathrm{GmbH}$ ) had a resonance frequency in the range between $\Omega$ $=76$ and $263 \mathrm{kHz}$ and a typical tip diameter below $50 \mathrm{~nm}$.

The AFM tip was illuminated by two different sources, depending on whether it was used for imaging or spectroscopy purposes. For near-field imaging, IR radiation from a continuous-wave grating-tuned $\mathrm{CO}_{2}$ gas laser (PL5, Edinburgh Instruments, UK) was employed. The power incident at the AFM system was set between 5 and $10 \mathrm{~mW}$. For nano-FTIR spectroscopy, broadband synchrotron radiation from the Metrology Light Source (MLS) was used. ${ }^{20,29}$ The MLS 
produces $25 \mathrm{ps}$ pulses at a repetition rate of $500 \mathrm{MHz}$ and provides ultrabroadband IR radiation with a time averaged power integrated over the spectral range from 400 to 4000 $\mathrm{cm}^{-1}$ of about $2 \mathrm{~mW}$. The MLS was operated in a special user mode, described in ref 28 , which enables the spectroscopy of thin organic layers.

Measured quantities are both the amplitude $s$ and phase $\phi$ of the IR radiation scattered from the Au-coated AFM probe via interferometric methods, ${ }^{6,30}$ referenced to a known material. In this case, a plain gold surface served as a reference. To extract the nanoscale-resolved electrical near-field component from the scattered field, $\eta=s \exp (\mathrm{i} \phi)$, higher harmonic demodulation technique was applied, ${ }^{31}$ obtaining $\eta_{n}=s_{n} \exp \left(\mathrm{i} \phi_{n}\right)$. Here, the detector signal was demodulated at higher harmonics $n$ of the tip modulation frequency $\Omega_{n}=n \Omega$.

IR reflection/absorption spectroscopy (IRRAS) was performed using a gold substrate to identify the adsorbed species. Before use, the Au surface was cleaned in bichromosulfuric acid and copious amounts of Milli-Q water $(18.2 \mathrm{M} \Omega \mathrm{cm})$. This cleaning procedure assured a completely hydrophilic gold surface. It is assumed that the island formation of $d$-DSPC and surfactin is created at the water surface in the LB trough, and hence, the shapes of the ML films on other substrates were similar to the films prepared on the gold substrate. However, some deviations may occur because of the different hydrophobicity/hydrophilicity of the substrates, as discussed below. The IRRAS measurements were carried out using a Bruker IFS66 spectrometer purged with nitrogen gas and equipped with a mercury cadmium telluride detector. The angle of incidence was $85^{\circ}$, the spectral resolution was $2 \mathrm{~cm}^{-1}$, and the spectrum was averaged over 3000 scans. The IR spectra of the corresponding pure chemicals ( $d$-DSPC and surfactin) in the powder form were acquired on a Bruker Tensor 37 system, using the attenuated total reflection (ATR) mode. The resolution was $4 \mathrm{~cm}^{-1}$, and the spectra were averaged over 1024 scans. The relative peaks heights in the IRRAS spectrum can be distorted because of the highly sloping background of the raw spectrum. Note that the spectra shown in Figure 2 have been baseline-corrected.

$d$-DSPC (1,2-distearoyl- $d_{70}$-sn-glycero-3-phosphocholine$1,1,2,2-d_{4}-N, N, N$-trimethyl- $d_{9}$ ) with a purity of $>99 \%$ was purchased from Avanti Polar Lipids. Surfactin (>98\%) and chloroform (99.8\%, stabilized with amylene) were purchased from Sigma-Aldrich. The compounds were used as received.

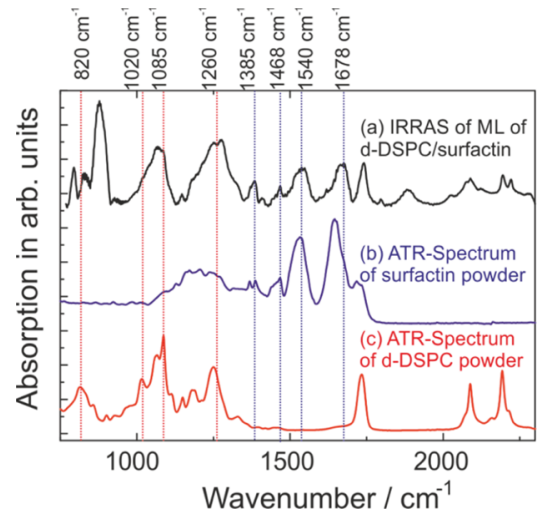

Figure 2. IRRAS spectrum [trace (a)] of the MLs on a Au substrate and absorption spectra (ATR) of $d$-DSPC and surfactin powder [trace $(b, c)$, respectively].
The molecular structures of surfactin and $d$-DSPC are shown in Figure 1 . The corresponding absorption spectra of powdered $d$ DSPC and surfactin samples are shown in Figure 2.

\section{RESULTS AND DISCUSSION}

To verify that $d$-DSPC and surfactin were adsorbed at the substrates, grazing incidence IRRAS was used to determine the spectrum of the $d$-DSPC/surfactin mixture adsorbed at a gold surface. The spectrum represented by trace (a) in Figure 2 shows a number of characteristic peaks originating from both, $d$-DSPC and surfactin. For $d$-DSPC, the symmetric and antisymmetric $\mathrm{PO}_{2}$ stretches are observed at 1085 and 1260 $\mathrm{cm}^{-1}, 32$ respectively, whereas the former is blended with the $\mathrm{CD}_{2}$ and $\mathrm{CD}_{3}$ bending vibrations. ${ }^{33}$ At $820 \mathrm{~cm}^{-1}$, the antisymmetric $\mathrm{O}-\mathrm{P}-\mathrm{O}$ stretch of $d$-DSPC shows a pronounced band, and in the region $2100-2300 \mathrm{~cm}^{-1}$, the $\mathrm{CD}_{2}$ and $\mathrm{CD}_{3}$ stretching vibrations are observed. For surfactin, the $\mathrm{CH}_{2}$ and $\mathrm{CH}_{3}$ deformation modes are observed at 1385 and $1468 \mathrm{~cm}^{-1,34}$ the amide II band is observed at $1540 \mathrm{~cm}^{-1}, 35$ the amide $\mathrm{I}$ band is observed at $1678 \mathrm{~cm}^{-1,35}$ and the $\mathrm{C}=\mathrm{O}$ stretch in both $d$-DSPC and surfactin is observed at $1740 \mathrm{~cm}^{-1}$, respectively. ${ }^{35,36}$ For the peaks at 890 and $1880 \mathrm{~cm}^{-1}$ observed for the $d$-DSPC/surfactin mixture, there seems no counterpart in the corresponding powder spectra. The peaks may be spectral artifacts, for example, due to the background, especially because there are no vibrations expected as high as $1880 \mathrm{~cm}^{-1}$, and their origin is currently unknown. However, all characteristic peaks originating from both compounds are observed and confirm their presence on the substrate.

The $d$-DSPC/surfactin mixture forms domains of submicrometer size, as shown in Figure 3. The topography image (a) shows the ML mixture deposited on the graphene sample and its typical terrace structures, which develop during the epitaxial growth of graphene on $\mathrm{SiC}^{37}$ The area captured by the image contains both uncovered (left part) and lipid/ surfactin-covered regions (right part) because it is centered at the position up to which the ML was deposited by the $\mathrm{LB}$ technique. The ML-covered areas can be clearly seen in the material-sensitive AFM-phase image depicted in Figure 3b. Here, the predominantly green regions correspond to the lipidand-surfactin-covered area, whereas the predominantly blue region shows the uncovered graphene ML.

Because the $\mathrm{SiC}$ terraces are atomically flat, the topography image allows the determination of the thickness of the lipid domains along the black and red arrows shown in Figure 3a, which are located on a single terrace. The black arrow runs from the plain substrate on top of the slightly thinner domain, from which a layer thickness of $(1.8 \pm 0.4) \mathrm{nm}$ can be deduced. The red arrow runs from the thicker domain to the thinner domain, resulting in a height difference of $(1.9 \pm 0.1) \mathrm{nm}$. The total thickness of the thicker layer can thus be derived as $(3.7 \pm$ $0.5) \mathrm{nm}$. The lower bound of this result of $3.2 \mathrm{~nm}$ still slightly differs from the expected theoretical thickness of $2.5 \mathrm{~nm}$. Note, however, that the AFM tapping mode has been employed, which, for changes in the material-related surface forces across a topographical step, is known to produce an additional uncertainty. ${ }^{38,39}$

The topography of the lipid mixture deposited on the Au substrate is shown in Figure 3c. The surface roughness of the $\mathrm{Au}$ substrate is of the same order of the domain thickness. Nevertheless, the domain geometry can be seen in the corresponding AFM-phase image in Figure 3d. Compared to the graphene substrate, the $\mathrm{Au}$ surface provides a sufficient 
(a)

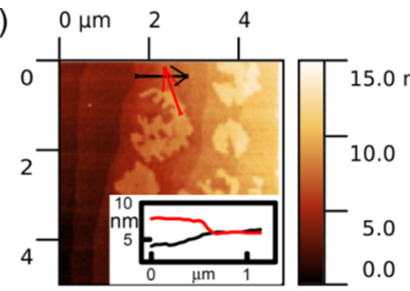

(c)

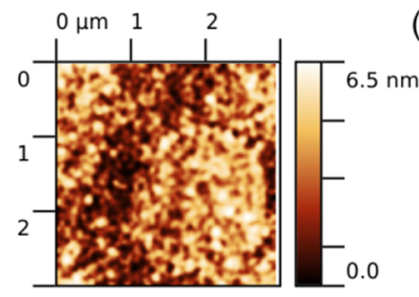

(b)

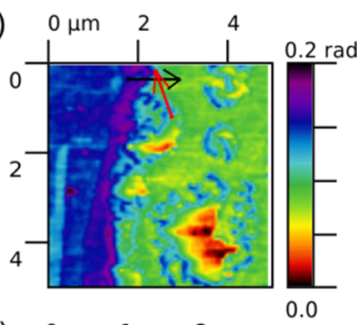

(d)

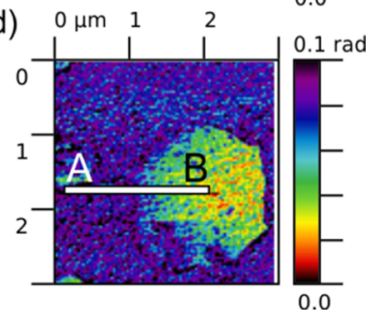

Figure 3. AFM images show (a) the topography and (b) the corresponding phase contrast of the deposited lipid domains on the epitaxially grown graphene ML. The area captured contains both uncovered and lipid-covered regions of the substrate. In the phase image, the predominantly green regions correspond to lipid-covered area, whereas the predominantly blue region shows the exposed graphene ML. The topography values along the two line cuts following the black and red arrows in (a) and (b) correspond to steps over different lipid domains and are shown in the inset of (a). (c) AFM topography and (d) the corresponding phase contrast of the lipid mixture deposited on Au substrate mapped with a higher magnification to capture a single domain. The region mapped is completely covered with the lipid mixture. The white horizontal line shown in (d) crosses the domain boundary. A sequence of 40 nano-FTIR spectra has been recorded along this line shown in Figure 4a.

enhancement of the near-field signal to allow the acquisition of nano-FTIR spectra from the ML. ${ }^{28}$ A line scan of locally resolved spectra will be discussed below and has been performed along the white horizontal line shown in Figure $3 \mathrm{~d}$, crossing a domain boundary. To determine the packing order of $d$-DSPC in this mixed system, vibrational sum frequency spectroscopy (VSFS) was used. As explained in more detail in the Supporting Information, it was concluded that the $d$-DSPC hydrocarbon chains possess a fairly ordered structure, although not in a perfect all-trans configuration. Thus, although surfactin is a bulky molecule, the $d$-DSPC islands are, on average, fairly ordered.

The results of the line scan are shown in Figure 4. It displays the phase part of the nano-FTIR spectrum varying along the white line in Figure 3d. The position $x=0 \mu \mathrm{m}$ corresponds to the side labeled as " $\mathrm{A}$ " and the position $x=2 \mu \mathrm{m}$ corresponds to the side labeled as "B" in Figure $3 \mathrm{~d}$. The domain boundary lies at the position $x=1 \mu \mathrm{m}$. The phase part of the s-SNOM signal, $\phi_{2}(\tilde{\nu})$, has been chosen because the near-field phase has been identified previously to correspond closely to the IR absorbance measured in the grazing incidence configuration. ${ }^{40}$ The near-field signal was obtained from locking onto the second harmonic of the oscillation frequency of the tip, $\Omega$, and referencing to the spectrum of a plain Au surface.

The line scan consists of 40 spectra recorded in steps of 50 $\mathrm{nm}$ over the spectral range of $780-1100 \mathrm{~cm}^{-1}$. Within this range, the combined tip enhancement and detector sensitivity lead to sufficient signal for the analysis of the individual, that is, nonaveraged spectra. Spectral features near 820 and $1030 \mathrm{~cm}^{-1}$ are visible at positions between $x=1$ and $2 \mu \mathrm{m}$, which, according to Figure $3 \mathrm{c}$,d, belong to the topographically higher

(a) Wavenumber $\tilde{\mathrm{v}}$ in $\mathrm{cm}^{-1}$
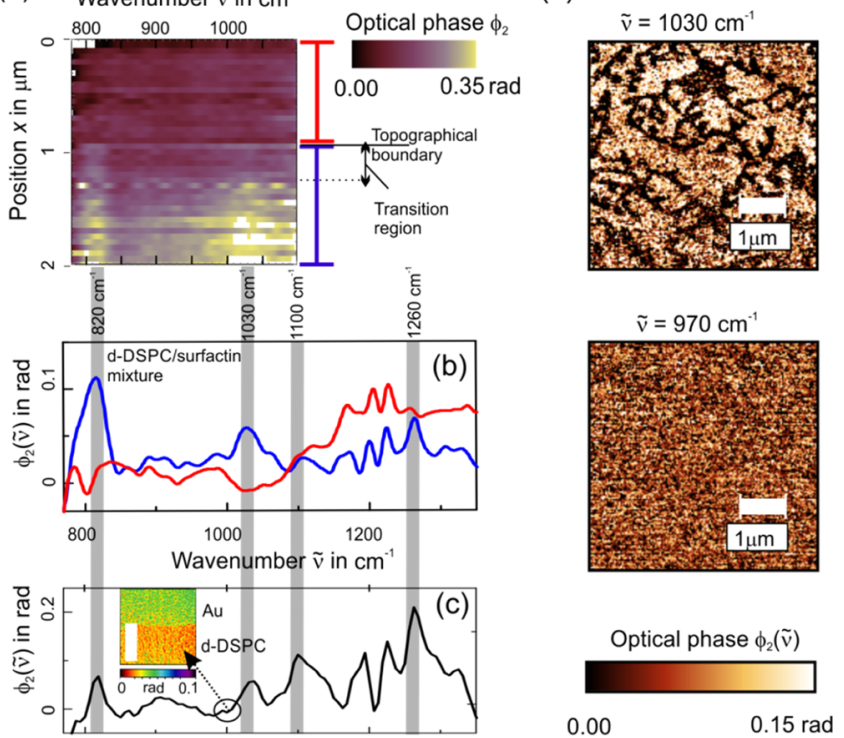

Figure 4. Nano-FTIR spectra of the $d$-DSPC/surfactin mixture on a $\mathrm{Au}$ substrate are shown in (a) as a function of the position along the white line marked in Figure $3 \mathrm{~d}$. The ends labeled "A" and "B" in Figure $3 \mathrm{~d}$ correspond to positions 0 and $2 \mu \mathrm{m}$ in (a), respectively. A transition region with reduced peak heights may be identified as marked in the figure. The spectra shown in (b) are matched to the wavenumber axis in (a). The spectra are averaged over the positions from 0 to $1 \mu \mathrm{m}$ (red) and 1 to $2 \mu \mathrm{m}$ (blue). For comparison, a nanoFTIR spectrum of a pure $d$-DSPC ML on a Au substrate is shown in (c). The gray areas indicate the prominent bands found in (b) and (c) and correspond to the features of $d$-DSPC shown in Figure 2. The inset shows an AFM mechanical phase plot with a white scale bar of 1 $\mu \mathrm{m}$ length. The $d$-DSPC layer forms a closed layer up to the position at which the ML was deposited by the LB technique. A twodimensional plot of the optical phase signal $\phi$ at two different laser frequencies is shown in $(d)$.

domain. A larger spectral range of $780-1350 \mathrm{~cm}^{-1}$ can be analyzed by averaging all spectra on either side of the domain boundary. The result is shown as the red and the blue curves in Figure $4 \mathrm{~b}$, corresponding to positions $x=0-1$ and $1-2 \mu \mathrm{m}$, respectively. All peaks marked by the gray bars are also found for $d$-DSPC in the ATR and IRRAS spectra (although these spectra have different spectral resolutions and exhibit slight shifts $^{40}$ ), as shown in Figure 2. The peaks near $1200 \mathrm{~cm}^{-1}$ may be artifacts as a result of the reduced intensity of the radiation source in this spectral region.

As a result, the above observations indicate that the topographically higher domain is mainly composed of $d$ DSPC. This conclusion is further supported by the near-field spectrum shown in Figure 4c. This spectrum corresponds to a sample on which only the $d$-DSPC layer has been deposited. Again, all features marked by the gray bars coincide with the spectrum of Figure $4 \mathrm{~b}$ indicated by blue color, belonging to the topographically higher domain. In the topographically lower domain, no $d$-DSPC content could be detected, allowing only surfactin to be the main constituent. Note that a stronger field enhancement at the AFM tip would be required for nano-FTIR measurements on the above samples for wavenumbers above $1300 \mathrm{~cm}^{-1}$, to achieve direct chemical identification of surfactin MLs.

For IR imaging of the spatial distribution of the $d$-DSPC ML, the tip was illuminated by monochromatic radiation at one of 
the resonant modes. Figure $4 \mathrm{~d}$ shows the corresponding map of the phase signal, $\phi_{2}$, at a wavenumber of $\tilde{\nu}=1030 \mathrm{~cm}^{-1}$, which was within the tuning range of the $\mathrm{CO}_{2}$ laser and also corresponds to one of the observed peaks in the nano-FTIR spectrum shown in Figure $4 \mathrm{~b}$. The contrast between the different domains in the upper image of Figure $4 \mathrm{~d}$ is clearly visible. Tuning the laser to $\tilde{\nu}=970 \mathrm{~cm}^{-1}$ where no resonant mode was observed results in a vanishing contrast, as shown in the lower part of Figure $4 \mathrm{~d}$.

In the above imaging investigations, the contrast resulted from small shifts in the optical phase produced by the approximately $3.7 \mathrm{~nm}$ thick $d$-DSPC film. This phase signal is reduced even further for thinner layers, such that the sensitivity to detect any $d$-DSPC content in the topographically lower domain is expected not to be sufficient. To further enhance the contrast for imaging thin films, it is proposed to use substrates which support the propagation of surface plasmons excited by a near-field probe. Here, epitaxial graphene on $\mathrm{SiC}$ is used as a substrate. Previously, it has been demonstrated that IR radiation focused on a sharp metalized tip as in the present sSNOM setup may launch circular surface plasmons in the graphene layer around the tip. ${ }^{41,42}$ The plasmon generation and the subsequent propagation are both sensitive to the wavenumber of the incident laser radiation $\tilde{\nu}_{\mathrm{L}}$ as well as to the dielectric environment of the graphene, described by the dielectric function of the $\mathrm{SiC}$ substrate, $\varepsilon_{1}$, and for the material on top of the graphene, $\varepsilon_{2}$. The surface plasmons may be reflected whenever the dielectric environment changes along the propagation path, leading to characteristic spatial interference patterns, for which it is considered as plasmon interferometry. ${ }^{43,44}$

In the present case, changes in the dielectric environment should mainly be caused by variations in the layer thickness of the lipid, its chemical composition, and its structure. Plasmon generation will also be sensitive to processes influencing the Fermi energy of the graphene, $E_{\mathrm{F}}$, such as the molecular orientation due to its dipole character. ${ }^{45,46}$ The resolution limit depends on the order of magnitude of the plasmon wavelength, $\lambda_{\mathrm{P}}$, as described by the simplified plasmon dispersion relation: ${ }^{47}$

$$
\lambda_{\mathrm{P}}=\frac{e^{2} E_{\mathrm{F}}}{2 \pi^{2} \hbar^{2} c^{2} \varepsilon_{0}\left(\varepsilon_{1}+\varepsilon_{2}\right)} \frac{1}{{\widetilde{\nu_{L}}}^{2}}
$$

where $\hbar$ is the reduced Planck constant, $c$ is the speed of light, and $\varepsilon_{0}$ is the vacuum permittivity. The interference pattern detected by the graphene layer constitutes an additional contrast mechanism on top of the near-field scattering on the tip-lipid system.

This method is illustrated in Figure 5. The topography of the lipid mixture containing a particularly large domain is shown in Figure 5a. It should be mentioned that the order and orientation of the molecules may differ from that on the gold surface because gold is hydrophilic, whereas graphene is more hydrophobic. However, clear domain formation is observed in both cases, which is of most relevance in this study. To resolve a potential plasmon standing wave pattern, the wavenumber of the laser radiation $\tilde{\nu}_{\mathrm{L}}$ should be sufficiently different from the longitudinal optical phonon frequency of the $\mathrm{SiC}$ substrate ${ }^{48}$ to avoid damping, that is, $\tilde{\nu}>950 \mathrm{~cm}^{-1}$. In addition, to prevent damping due to lipid ML absorption, one requires $\tilde{\nu}<1000$ $\mathrm{cm}^{-1}$, such that for the measurement in Figure $5 \mathrm{~b}$, the wavenumber was set to $\tilde{\nu}=978 \mathrm{~cm}^{-1}$. The maps in Figure 5 present the scattering amplitude $s$ showing, on average, a higher

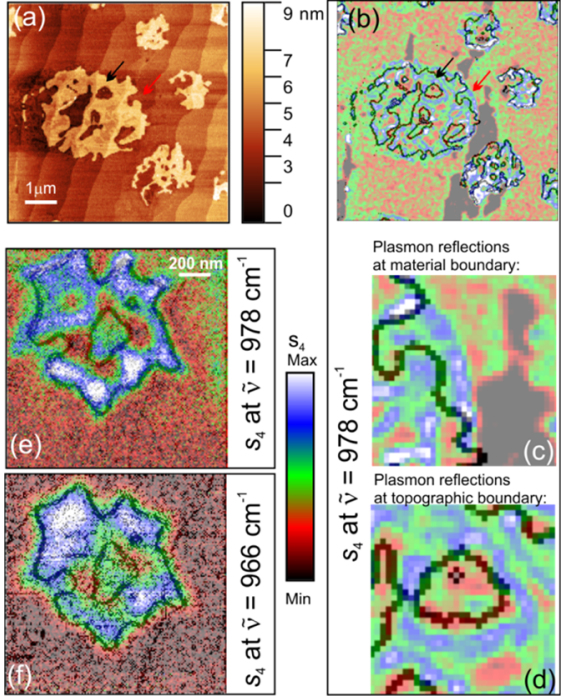

Figure 5. $d$-DSPC/surfactin ML domains on an epitaxial graphene substrate. The AFM topography is shown in (a). Optical signal amplitude demodulated at the fourth harmonic, $s_{4}$, at $\tilde{\nu}=978 \mathrm{~cm}^{-1}$ is plotted in (b). The black lines represent the edges of the domains. The optical contrast deviates from the topographical data, with two examples marked by the two arrows shown in (a) and (b). The corresponding magnified areas are shown in (c) and (d) for the red and black arrows, respectively. (c) (red arrow) Example of contrast over a topographically flat region, clearly showing the material boundary; (d) (black arrow) contrast around topographical boundaries. A smaller domain [not shown in (a)] is plotted in (e) and (f), imaged at $\tilde{\nu}=966$ and $978 \mathrm{~cm}^{-1}$, respectively. The feature size is of the order of the estimated plasmon wavelength $\lambda_{\mathrm{P}}$, for which the contrast becomes sensitive to small changes in $\tilde{\nu}$ and hence $\lambda_{\mathrm{P}}$ in (e) and (f).

scattering probability (blue color code) over the topographically higher domain, together with a fine structure discussed below. In the dark regions, $s$ is minimal as a result of unwanted bilayer graphene domains. ${ }^{49}$ The topographical edges of the domain appearing as black lines are superimposed over the scattering amplitude $s$.

In some regions, the interference pattern deviates from the edges defined by the topography, indicating additional materialrelated boundaries. Two examples are illustrated by the red and black arrows in Figure 5a,b. The corresponding magnified areas are shown in Figure $5 \mathrm{c}$,d. In the first example, interference stripes along a topographically flat region are visible in (c). In this case, plasmon reflections may have occurred at a material boundary, such as between a surfactin and a $d$-DSPC-rich domain. The above measurements shown in Figure 4 indicate that the $d$-DSPC-rich domain is about $2-3 \mathrm{~nm}$ higher, whereas in the case shown in Figure 5, they are of the same height. This may be due to a collapse of the $d$-DSPC molecules in this border region and a resulting loss of ordering. A signature of this border region may also be visible in the line scan in Figure 4a. A transition region may be identified within $x=1.0-1.3 \mu \mathrm{m}$ in this line scan, as indicated in Figure 4a. In this region, the peak height is significantly reduced, which may be due to a reduced thickness.

The second example depicted in Figure $5 \mathrm{~d}$ shows an interference pattern caused by topographical boundaries. An abrupt change in the thickness will also lead to a change in the dielectric environment. Hence, an interference pattern due to 
topographical boundaries can also be expected in a chemically homogeneous area.

An example of a particularly small domain is shown in Figure 5e,f at laser wavenumbers of $\tilde{\nu}=978 \mathrm{~cm}^{-1}$ (as above) and 966 $\mathrm{cm}^{-1}$, respectively. The Fermi energy of the graphene before being covered by the lipid was $E_{\mathrm{F}} \approx 200 \mathrm{meV}$ so that $\tilde{\nu}=978$ $\mathrm{cm}^{-1}$ is still sufficiently low to avoid interband absorption in the graphene layer at $2 E_{\mathrm{F}}$. From eq 1 , one derives for uncovered graphene $\lambda_{\mathrm{P}} \approx 370 \mathrm{~nm}$ for laser wavenumbers of $\tilde{\nu}=978 \mathrm{~cm}^{-1}$ and $\lambda_{\mathrm{P}} \approx 600 \mathrm{~nm}$ for $\tilde{\nu}=966 \mathrm{~cm}^{-1}$. Hence, in the latter case, the plasmon wavelength, $\lambda_{\mathrm{P}}$, is closer to the order of the feature size and accordingly less of this structure can be resolved. This effect is confirmed in Figure 5e,f. Whereas in Figure 5e, the upper left part of the domain still contains details on some parts of the domain structure, in Figure $5 \mathrm{f}$, these details evidently disappear. Note that $\lambda_{\mathrm{P}}$ grows rapidly as $\tilde{\nu}$ approaches the $\mathrm{SiC}$ phonon resonance where $\varepsilon_{1}$ approaches zero.

\section{CONCLUSIONS}

We have employed near-field FTIR microscopy and spectroscopy in combination with AFM imaging to study the domain structure of mixed MLs of the biologically relevant phospholipid DSPC in the deuterated form and the antibiotic surfactin. The obtained chemical information enabled the investigation of the domain formation of the two components with a spatial resolution of $50 \mathrm{~nm}$. Furthermore, a novel method to enhance the near-field imaging contrast by plasmon interferometry using graphene-covered substrates was demonstrated. Scanning thin organic layers on top of graphene at appropriate doping levels is demonstrated to be a contrastenhancing method for detecting material and molecular orientation changes, influencing the dielectric environment and doping properties of graphene, respectively. The concept was successfully demonstrated by revealing a transition region of the domain boundaries of the organic MLs. The combined spectroscopic and topographic information of domains of biomolecules could thus be used to investigate the corresponding interaction mechanisms. Such a knowledge is of great importance in furthering our understanding about the complex structure of cell membranes as well as in determining the interaction of drugs on cell membranes at the nanolevel.

\section{ASSOCIATED CONTENT}

\section{S Supporting Information}

The Supporting Information is available free of charge on the ACS Publications website at DOI: 10.1021/acsomega.7b01931.

VSFS of $d$-DSPC and surfactin on a gold surface for the investigation of the packing order of $d$-DSPC (PDF)

\section{AUTHOR INFORMATION}

\section{Corresponding Author}

*E-mail: Bernd.Kaestner@ptb.de (B.K.).

\section{ORCID}

Bernd Kästner: 0000-0002-6575-6621

Eckart Rühl: 0000-0002-0451-8734

\section{Present Addresses}

${ }^{\perp}$ West Pharmaceutical Services Deutschland GmbH \& Co. KG, Stolberger Straße 21-41, 52249 Eschweiler, Germany (P.H.).

${ }^{\#}$ National Institute of Standards and Technology (NIST), 100 Bureau Drive, MD 20899, USA (M.K.).

\section{Funding}

This project has received funding from the EMRP and EMPIR programme cofinanced by the Participating States and from the European Union's Horizon 2020 Research and Innovation Programme. Financial support by SFB 1112 (project B02) is gratefully acknowledged. M.K. gratefully acknowledges the support by the Braunschweig International Graduate School of Metrology (B-IGSM) and NanoMet.

\section{Notes}

The authors declare no competing financial interest.

\section{ACKNOWLEDGMENTS}

We gratefully acknowledge discussion with Vladimir A. Zenin and Alexander A. Govyadinov. We thank Thomas Ederth at Linköping University (Sweden) for help with the IRRAS spectra and Eric Tyrode at KTH (Sweden) for support with the VSF spectra.

\section{REFERENCES}

(1) Brown, D. A.; London, E. Structure and Origin of Ordered Lipid Domains in Biological Membranes. J. Membr. Biol. 1998, 164, 103114.

(2) Brown, D. A.; Rose, J. K. Sorting of GPI-anchored proteins to glycolipid-enriched membrane subdomains during transport to the apical cell surface. Cell 1992, 68, 533-544.

(3) Pike, L. J. Lipid rafts: bringing order to chaos. J. Lipid Res. 2003, 44, 655-667.

(4) Born, M.; Wolf, E. Principles of Optics: Electromagnetic Theory of Propagation, Interference and Diffraction of Light, 7th ed.; Cambridge University Press: Cambridge, 2002.

(5) Levin, P. A.; Angert, E. R. Small but Mighty: Cell Size and Bacteria. Cold Spring Harbor Perspect. Biol. 2015, 7, a019216.

(6) Huth, F.; Govyadinov, A.; Amarie, S.; Nuansing, W.; Keilmann, F.; Hillenbrand, R. Nano-FTIR absorption spectroscopy of molecular fingerprints at $20 \mathrm{~nm}$ spatial resolution. Nano Lett. 2012, 12, 39733978.

(7) Toseland, C. P. Fluorescent labeling and modification of proteins. J. Chem. Biol. 2013, 6, 85-95.

(8) Amenabar, I.; Poly, S.; Nuansing, W.; Hubrich, E. H.; Govyadinov, A. A.; Huth, F.; Krutokhvostov, R.; Zhang, L.; Knez, M.; Heberle, J.; Bittner, A. M.; Hillenbrand, R. Structural analysis and mapping of individual protein complexes by infrared nanospectroscopy. Nat. Commun. 2013, 4, 2890.

(9) Berweger, S.; Nguyen, D. M.; Muller, E. A.; Bechtel, H. A.; Perkins, T. T.; Raschke, M. B. Nano-chemical infrared imaging of membrane proteins in lipid bilayers. J. Am. Chem. Soc. 2013, 135, $18292-18295$.

(10) Giliberti, V.; Baldassarre, L.; Rosa, A.; de Turris, V.; Ortolani, M.; Calvani, P.; Nucara, A. Protein clustering in chemically stressed HeLa cells studied by infrared nanospectroscopy. Nanoscale 2016, 8, $17560-17567$.

(11) Khatib, O.; Keilmann, F.; McLeod, A. S.; Basov, D. N.; Wood, J. D.; McLeod, A. S.; Goldflam, M. D.; Wagner, M.; Damhorst, G. L.; Koepke, J. C.; Doidge, G. P.; Rangarajan, A.; Bashir, R.; Pop, E.; Lyding, J. W.; Thiemens, M. H.; Basov, D. N. Graphene-Based Platform for Infrared Near-Field Nanospectroscopy of Water and Biological Materials in an Aqueous Environment. ACS Nano 2015, 9, $7968-7975$.

(12) Brehm, M.; Taubner, T.; Hillenbrand, R.; Keilmann, F. Infrared spectroscopic mapping of single nanoparticles and viruses at nanoscale resolution. Nano Lett. 2006, 6, 1307-1310.

(13) Vitry, P.; Rebois, R.; Bourillot, E.; Deniset-Besseau, A.; Virolle, M.-J.; Lesniewska, E.; Dazzi, A. Combining infrared and mode synthesizing atomic force microscopy: Application to the study of lipid vesicles inside Streptomyces bacteria. Nano Res. 2016, 9, 1674-1681. 
(14) Amarie, S.; Zaslansky, P.; Kajihara, Y.; Griesshaber, E.; Schmahl, W. W.; Keilmann, F. Nano-FTIR chemical mapping of minerals in biological materials. Beilstein J. Nanotechnol. 2012, 3, 312-323.

(15) Lu, F.; Jin, M.; Belkin, M. A. Tip-enhanced infrared nanospectroscopy via molecular expansion force detection. Nat. Photonics 2014, 8, 307-312.

(16) Nagle, J. F.; Tristram-Nagle, S. Structure of lipid bilayers. Biochim. Biophys. Acta, Rev. Biomembr. 2000, 1469, 159-195.

(17) Sheppard, J. D.; Jumarie, C.; Cooper, D. G.; Laprade, R. Ionic channels induced by surfactin in planar lipid bilayer membranes. Biochim. Biophys. Acta, Rev. Biomembr. 1991, 1064, 13-23.

(18) Maget-Dana, R.; Ptak, M. Interactions of surfactin with membrane models. Biophys. J. 1995, 68, 1937-1943.

(19) Bouffioux, O.; Berquand, A.; Eeman, M.; Paquot, M.; Dufrêne, Y. F.; Brasseur, R.; Deleu, M. Molecular organization of surfactinphospholipid monolayers: effect of phospholipid chain length and polar head. Biochim. Biophys. Acta, Biomembr. 2007, 1768, 1758-1768.

(20) Hermann, P.; Hoehl, A.; Patoka, P.; Huth, F.; Rühl, E.; Ulm, G. Near-field imaging and nano-Fourier-transform infrared spectroscopy using broadband synchrotron radiation. Opt. Express 2013, 21, 29132919.

(21) Hermann, P.; Hoehl, A.; Ulrich, G.; Fleischmann, C.; Hermelink, A.; Kästner, B.; Patoka, P.; Hornemann, A.; Beckhoff, B.; Rühl, E.; Ulm, G. Characterization of semiconductor materials using synchrotron radiation-based near-field infrared microscopy and nanoFTIR spectroscopy. Opt. Express 2014, 22, 17948.

(22) Bechtel, H. A.; Muller, E. A.; Olmon, R. L.; Martin, M. C.; Raschke, M. B. Ultrabroadband infrared nanospectroscopic imaging. Proc. Natl. Acad. Sci. U.S.A. 2014, 111, 7191-7196.

(23) Pollard, B.; Maia, F. C. B.; Raschke, M. B.; Freitas, R. O. Infrared Vibrational Nanospectroscopy by Self-Referenced Interferometry. Nano Lett. 2016, 16, 55-61.

(24) Demel, R. A.; van Kessel, W. S. M. G.; Zwaal, R. F. A.; Roelofsen, B.; van Deenen, L. L. M. Relation between various phospholipase actions on human red cell membranes and the interfacial phospholipid pressure in monolayers. Biochim. Biophys. Acta, Rev. Biomembr. 1975, 406, 97-107.

(25) Li, P.; Wang, T.; Böckmann, H.; Taubner, T. GrapheneEnhanced Infrared Near-Field Microscopy. Nano Lett. 2014, 14, 4400-4405.

(26) Li, P.; Taubner, T. Broadband subwavelength imaging using a tunable graphene-lens. ACS Nano 2012, 6, 10107-10114.

(27) Kruskopf, M.; Pakdehi, D. M.; Pierz, K.; Wundrack, S.; Stosch, R.; Dziomba, T.; Götz, M.; Baringhaus, J.; Aprojanz, J.; Tegenkamp, C.; Lidzba, J.; Seyller, T.; Hohls, F.; Ahlers, F. J.; Schumacher, H. W. Comeback of epitaxial graphene for electronics: large-area growth of bilayer-free graphene on SiC. 2D Mater. 2016, 3, 41002.

(28) Hermann, P.; Kästner, B.; Hoehl, A.; Kashcheyevs, V.; Patoka, P.; Ulrich, G.; Feikes, J.; Ries, M.; Tydecks, T.; Beckhoff, B.; Rühl, E.; Ulm, G. Enhancing the sensitivity of nano-FTIR spectroscopy. Opt. Express 2017, 25, 16574-16588.

(29) Gottwald, A.; Klein, R.; Müller, R.; Richter, M.; Scholze, F.; Thornagel, R; Ulm, G. Current capabilities at the Metrology Light Source. Metrologia 2012, 49, S146-S151.

(30) Ocelic, N.; Huber, A.; Hillenbrand, R. Pseudoheterodyne detection for background-free near-field spectroscopy. Appl. Phys. Lett. 2006, 89, 101124.

(31) Hillenbrand, R.; Keilmann, F. Complex optical constants on a subwavelength scale. Phys. Rev. Lett. 2000, 85, 3029-3032.

(32) Tamm, L. K.; Tatulian, S. A. Infrared spectroscopy of proteins and peptides in lipid bilayers. Q. Rev. Biophys. 1997, 30, 365-429.

(33) Liang, C. Y.; Pearson, F. G. Infrared spectra of crystalline and stereoregular polymers. J. Mol. Spectrosc. 1961, 5, 290-306.

(34) Jung, J.; Yu, K. O.; Ramzi, A. B.; Choe, S. H.; Kim, S. W.; Han, S. O. Improvement of surfactin production in Bacillus subtilis using synthetic wastewater by overexpression of specific extracellular signaling peptides, comX and phrC. Biotechnol. Bioeng. 2012, 109, 2349-2356.
(35) Ferré, G.; Besson, F.; Buchet, R. Conformational studies of the cyclic 1, d-lipopeptide surfactin by Fourier transform infrared spectroscopy. Spectrochim. Acta, Part A 1997, 53, 623-635.

(36) Colthup, N. B.; Daly, L. H.; Wiberley, S. E. Introduction to Infrared and Raman Spectroscopy, 3rd ed.; Academic Press: London, 1990.

(37) Kruskopf, M.; Pierz, K.; Wundrack, S.; Stosch, R.; Dziomba, T.; Kalmbach, C.-C.; Müller, A.; Baringhaus, J.; Tegenkamp, C.; Ahlers, F. J.; Schumacher, H. W. Epitaxial graphene on SiC: modification of structural and electron transport properties by substrate pretreatment. J. Phys.: Condens. Matter 2015, 27, 185303.

(38) Nemes-Incze, P.; Osváth, Z.; Kamarás, K.; Biró, L. P. Anomalies in thickness measurements of graphene and few layer graphite crystals by tapping mode atomic force microscopy. Carbon 2008, 46, 14351442.

(39) Ukraintsev, E.; Kromka, A.; Kozak, H.; Remeš, Z.; Rezek, B. Atomic Force Microscopy Investigations into Biology-From Cell to Protein; Frewin, C., Ed.; InTech, 2012; ISBN 978-953-51-0114-7.

(40) Mastel, S.; Govyadinov, A. A.; de Oliveira, T. V. A. G.; Amenabar, I.; Hillenbrand, R. Nanoscale-resolved chemical identification of thin organic films using infrared near-field spectroscopy and standard Fourier transform infrared references. Appl. Phys. Lett. 2015, $106,23113$.

(41) Fei, Z.; Rodin, A. S.; Andreev, G. O.; Bao, W.; McLeod, A. S.; Wagner, M.; Zhang, L. M.; Zhao, Z.; Thiemens, M.; Dominguez, G.; Fogler, M. M.; Neto, A. H. C.; Lau, C. N.; Keilmann, F.; Basov, D. N. Gate-tuning of graphene plasmons revealed by infrared nano-imaging. Nature 2012, 487, 82-85.

(42) Chen, J.; Badioli, M.; Alonso-González, P.; Thongrattanasiri, S.; Huth, F.; Osmond, J.; Spasenović, M.; Centeno, A.; Pesquera, A.; Godignon, P.; Elorza, A. Z.; Camara, N.; de Abajo, F. J. G.; Hillenbrand, R.; Koppens, F. H. L. Optical nano-imaging of gatetunable graphene plasmons. Nature 2012, 487, 77-81.

(43) Fei, Z.; Rodin, A. S.; Gannett, W.; Dai, S.; Regan, W.; Wagner, M.; Liu, M. K.; McLeod, A. S.; Dominguez, G.; Thiemens, M.; Neto, A. H. C.; Keilmann, F.; Zettl, A.; Hillenbrand, R.; Fogler, M. M.; Basov, D. N. Electronic and plasmonic phenomena at graphene grain boundaries. Nat. Nanotechnol. 2013, 8, 821-825.

(44) Gerber, J. A.; Berweger, S.; O'Callahan, B. T.; Raschke, M. B. Phase-Resolved Surface Plasmon Interferometry of Graphene. Phys. Rev. Lett. 2014, 113, 55502.

(45) Wehling, T. O.; Katsnelson, M. I.; Lichtenstein, A. I. Adsorbates on graphene: Impurity states and electron scattering. Chem. Phys. Lett. 2009, 476, 125-134.

(46) Zhang, Y.; Brar, V. W.; Girit, C.; Zettl, A.; Crommie, M. F. Origin of spatial charge inhomogeneity in graphene. Nat. Phys. 2009, $5,722-726$.

(47) Jablan, M.; Buljan, H.; Soljačić, M. Plasmonics in graphene at infrared frequencies. Phys. Rev. B: Condens. Matter Mater. Phys. 2009, 80,245435

(48) Hillenbrand, R.; Taubner, T.; Keilmann, F. Phonon-enhanced light matter interaction at the nanometre scale. Nature 2002, 418, 159-162.

(49) Fei, Z.; Iwinski, E. G.; Ni, G. X.; Zhang, L. M.; Bao, W.; Rodin, A. S.; Lee, Y.; Wagner, M.; Liu, M. K.; Dai, S.; Goldflam, M. D.; Thiemens, M.; Keilmann, F.; Lau, C. N.; Castro-Neto, A. H.; Fogler, M. M.; Basov, D. N. Tunneling Plasmonics in Bilayer Graphene. Nano Lett. 2015, 15, 4973-4978. 